\title{
AN ELEMENTARY TAUBERIAN THEOREM OF NONLINEAR TYPE
}

\author{
HARRY POLLARD AND D. G. SAARI
}

Abstract. We find a nonlinear condition on $f \in C^{2}(0, \infty)$ which, when coupled with the assumption $\lim \inf |f(t)| / t<\infty$, implies $f \sim A t$ and $f^{\prime} \rightarrow A$. $A$ is some constant and $t \rightarrow \infty$ or $t \rightarrow 0+$.

In what follows the function $y(t)$ is of class $C^{\prime}(0, \infty), \omega$ is positive and measurable on $(-\infty, \infty)$, and $\phi$ is measurable on $(0, \infty)$. The motivation for the kind of theorems that follow is given by Saari [1], and Theorem B represents an improvement of his Theorems 1 and 2 .

The results are true whether $t \rightarrow \infty$ or $t \rightarrow 0+$ provided they are read consistently throughout.

Theorem A. If

$$
a \equiv \lim \inf y<\infty \quad \text { and } \quad A \equiv \lim \sup y>-\infty
$$

and

$$
y^{\prime}(t) \leqq \omega(y(t)) \phi(t),
$$

where $\phi$ is integrable, then $\lim y$ exists and is finite.

Proof. Choose two sequences $t_{n}, \tau_{n}, t_{n} \geqq \tau_{n}$, both approaching $\infty$ [or both approaching 0 ] so that $Y_{n} \equiv y\left(t_{n}\right) \rightarrow A, y_{n} \equiv y\left(\tau_{n}\right) \rightarrow a$. By (2) and the positivity of $\omega$

$$
\int_{y_{n}}^{Y_{n}} \frac{d y}{\omega(y)} \leqq \int_{\tau_{n}}^{t_{n}} \phi(\tau) d \tau .
$$

Let $n \rightarrow \infty$. Since $\phi$ is integrable, the right-hand side approaches zero.

Therefore $\int_{a}^{A}(d y / \omega(y)) \leqq 0$. Again because $\omega(y)>0$ and $A \geqq a$ this is possible only if $A=a=\infty$; or $A=a=-\infty$; or $A=a$, finite. The first two possibilities are ruled out by (1). This completes the proof.

Theorem B. Let $g \in C^{2}(0, \infty)$. If

$$
\lim \inf |g(t)| / t<\infty
$$

and

$$
g^{\prime \prime}(t) \leqq \omega\left(g^{\prime}(t)\right) \phi(t),
$$

Received by the editors July 15, 1969.

AMS Subject Classifications. Primary 2670, 2640, 4042; Secondary 3440.

Key Words and Phrases. Tauberian Theorems, Asymptotic behavior of derivatives. 
where $\phi$ is integrable, then the limits

$$
\lim g^{\prime}(t), \quad \lim g(t) / t
$$

exist and are equal.

Proof. Let $y=g^{\prime}(t)$. Then ( $\left.1^{\prime}\right)$ implies (1). For if $a=\infty$, then $g^{\prime} \rightarrow \infty, g(t) / t \rightarrow \infty$, a violation of $\left(1^{\prime}\right)$; and similarly if $A=-\infty$. Moreover ( $\left.2^{\prime}\right)$ is just (2). So by Theorem A we can conclude that $A=\lim g^{\prime}$ exists. It follows in turn that $\lim g(t) / t$ exists and equals $A$.

Theorem B improves Saari's theorems this way: in place of $\left(1^{\prime}\right)$ he requires $g(t)=O(t)$; in $\left(2^{\prime}\right)$ he requires $\phi \geqq 0$; and his conclusion is $g^{\prime}(t)=O(1)$.

\section{REFERENCE}

1. D. G. Saari, Some large $O$ nonlinear Tauberian theorems, Proc. Amer. Math. Soc. 21 (1969), 459-462.

Purdue University 